\title{
Compressing Turbulence Effect in FSO using New Modulation Technique
}

\author{
Norizan Mohamed Nawawi ${ }^{1,}$, Anuar Mat Safar ${ }^{1}$, Abdul Rahman Kram ${ }^{1}$, C.B.M. Rashidi ${ }^{1}$ \\ and Junita Mohd Nordin ${ }^{1}$ \\ ${ }^{1}$ Optical Research Group, Advanced Communication Engineering Centre of Excellence (ACE-COE), \\ School of Computer \& Communication Engineering, Universiti Malaysia Perlis (UNIMAP), \\ Malaysia.
}

\begin{abstract}
Diffusers transmitter modulation to reduce the effect of atmospheric turbulence in free space optical communication system is described in this paper. This technique uses dual transmitters and dual receivers which are using differential mode for detection. The combination of these components produce superior modulation method especially to reduce scintillation index, to overcome signal detection with fix zero threshold and improve power received. In order to improve the performance of free space optical system, these three elements play an important role. The analysis result show that for receiving power dual diffuser modulation at $3 \mathrm{~km}$ distance propagation is $4.59 \mathrm{dBm}$ compared to conventional OOK using diffuser which only $-7.6 \mathrm{dBm}$ and equivalent to $3 \mathrm{dBm}$ improvement or up to 40 percent better.
\end{abstract}

\section{Introduction}

Optical communication through free space is an attractive alternative over fiber optical communication where provides high bandwidth, fast-installation and high security [1]. However free space optic is suffering with the atmosphere disturbance which can lead the laser beam fading when reach the detector. Meanwhile, beam fading is a condition where the beam spread greater than diffraction estimated limit. Moreover, the front phase of the beam may varies and resulting intensity signal fluctuation. Taking account all these effect will result spatial and temporal random changes in refractive index of several elements such as temperature, pressure and wind along the optical propagation path [2-9].

Recently, phase screen diffuser which generates partial coherent beam has rising interest especially to improve the performance in laser communication system [10-13]. The original FSO employs the perfect coherence beam suffers some problems depends on weather condition. Propagation phenomena in the air such as scattering, absorption and turbulence spread the laser beam. In this paper we concern on atmospheric turbulence issue in the transmission system. Beam wander and redistribution of the intensity within the beam, phase-front distortion and beam broadening are three critical effects on the laser beam. The

* Corresponding author: norizan1123@gmail.com 
scintillation or temporary redistribution of the intensity, is the outcome from the chaotic fluctuation an atmosphere and from thermal gradients within the optical channel. Consequently, these condition can cause signal fading that will degrade the FSO performance.

We demonstrate the alternative method in order to diminish these effect, which using the phase screen diffuser device. This technique creates a 'new' Gaussian beam characteristic which effectively propagates through atmospheric turbulence. Partially coherent beam can enhance the performance of FSO system by applying two diffusers at transmitter and at receiver employs reception in differential mode detection technique. The fundamental of partial coherent beam can be found in next section followed by demonstration of proposed system model and then result and discussion. Last but not least, the final section is for conclusion

\section{Diffusers transmitter system model}

The proposed system uses On Off Keying (OOK) modulation to modulate the signal as reference for conventional system. It employs two transmitter, when the transmitter A sends a bit ' 1 ', the transmitter B will send the bit ' 0 ' (transmitter B is set in compliment condition) in simultaneously and vice versa. Immediately the signals will go through the subtractor for the differential mode detection process. This occurred at receiver as shown in Figure 1. Subtractor were assume as an ideal subtractor where zero signal loss during this process. Therefore, the output signal is bit ' 1 ' for input signal binary ' 1 ' and bit '- 1 ' for input signal binary ' 0 '. Table 1 illustrate the binary transmitting procedure. Here we can see that, the modulation approach eliminated the need of adaptive threshold where the conventional OOK modulation depends on threshold detection to recognize the incoming signal received whether the bit ' 1 ' or ' 0 '.

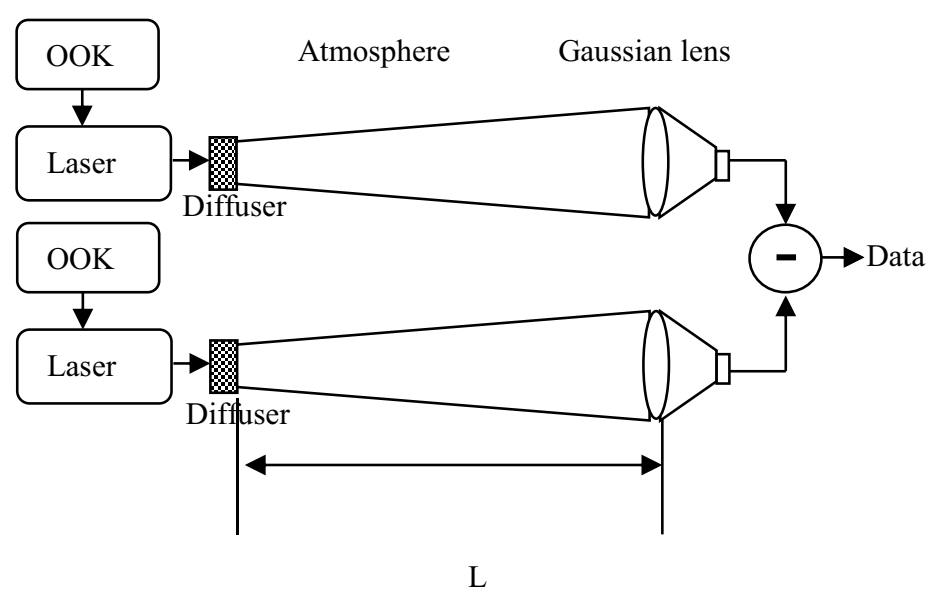

Fig. 1. Diffusers Modulation Setup

The conventional detection technique always misinterpretation by receiver where the noise alone capable to trigger as signal bit '1' (sending pulse) which also well known as 'false alarm' and sometimes the signal sending which contain the data is recognize as bit ' 0 ' (no pulse condition) when the signal is not reach the threshold value [17]. This condition is well known as 'miss detection' condition. Hence, the complex adaptive threshold has been suggested to overcome this problem. Whereas by employing dual diffuser modulation the 
detection signal is more precise with fix zero threshold. The incoming signal can be referenced to determine the input signal binary ' 1 ' or ' 0 '. Additionally, we placed the low cost material phase screen diffuser at transmitter to combat the atmospheric turbulence effect more efficient then give the optimum performance for overall system. This combination creates the superior robust modulation in FSOC. According to Table 1, let say $Y n$ is the received signal with zero threshold detection,

$$
\left.\begin{array}{l}
Y n>1 \\
Y n<1
\end{array}\right\} \begin{aligned}
& \text { bit ' } 1 ' \\
& \text { bit ' } 0 '
\end{aligned}
$$

The received signal can be written as:

$$
Y n=2 \sqrt{E_{b}}+\frac{n_{1}+n_{2}}{2}
$$

where $\sqrt{E_{b}}$ is represent the average energy signal and $n_{1}$ and $n_{2}$ are representing the noise of photo detector 1 and 2 respectively, where $\mathrm{n}$ can be denoted as Additive White Gaussian Noise (AWGN) with zero mean and variance $\sigma$.

Table 1. Transmit and Receive bits using Diffusers Modulation Approach

\begin{tabular}{|c|c|c|c|c|c|}
\hline \multicolumn{2}{|c|}{} & \multicolumn{2}{c|}{ Receiver (Rx) } & \multicolumn{2}{c|}{ Subtraction Process } \\
\hline Transmitter (Tx) & \multicolumn{2}{|c|}{ Received bit } & \multicolumn{2}{|c|}{ Rx A - Rx B } \\
\hline Sending bit & 1 & \multirow{2}{*}{ Rx A } & 1 & \multirow{2}{*}{ Bit 1} & 1 \\
\cline { 6 - 7 } \cline { 6 - 7 } & 0 & & 0 & \\
\hline Tx A & 0 & Rx B & 0 & Bit 0 & -1 \\
\hline
\end{tabular}

\section{Partially coherent beam beam}

Expressions for partially coherent beam are derived as same as a coherent beam [15] equation. The typical value for the diffuser correlation length $\left(l_{\mathrm{c}}^{2}\right)$ are $0.1,0.01,0.001$ and 0.0001 . Using the Kolmogorov spectrum and standard, extended Rytov theory on axis scintillation index for weak turbulence (inner scale $l=0$, Outer scale $L=\infty$ ) partially coherent Gaussian-beam is given by,

$$
\sigma_{B}^{2}=3.86 \sigma_{I}^{2}\left\{\begin{array}{l}
0.4\left[\left(1+2 \Theta_{e d}\right)^{2}+4\left(\Lambda_{e d}\right)^{2}\right]^{5 / 12} \\
\times\left(\cos \left[\frac{5}{6} \tan ^{-1}\left(\frac{1+2 \Theta_{e d}}{2 \Lambda_{e d}}\right)\right]\right) \\
-\left(\frac{11}{6}\left(\Lambda_{e d}\right)^{5 / 6}\right)
\end{array}\right\}
$$


where $\sigma_{I}^{2}$ indicate the strength of intensity fluctuations and proportional to Rytov variance as

$$
\sigma_{R}^{2}=1.23 C_{n}^{2} k^{7 / 6} L^{11 / 6}
$$

For weak fluctuation, it is less than 1 and for strong fluctuation it is greater than $1 . C_{n}^{2}$ is the refractive index structure constant that characterizes the strength of the index of refraction fluctuations. The typical $C_{n}^{2}$ value weak turbulence is $10^{-17} \mathrm{~m}^{-2 / 3}$ and strong $10^{-}$ ${ }^{12} \mathrm{~m}^{-2 / 3}$.

\subsection{Standard and effective beam parameters}

The effect of diffuser on an optical beam at the receiver is characterized by replacing the standard beam parameter $\Theta_{1}, \Lambda_{1}$ by effective beam parameter $\Theta_{e d}, \Lambda_{e d}$ define in term of $N_{\mathrm{s}}$ as follows

$$
\Lambda_{e d}=\frac{\Lambda_{0} \Lambda_{s}}{\Theta_{0}^{2}+\Lambda_{0}^{2} N_{S}}
$$

and

$$
\Theta_{e d}=\frac{\Theta_{0}}{\Theta_{0}^{2}+\Lambda_{0}^{2} N_{S}}
$$

where

$$
\Lambda_{1}=\frac{\Lambda_{0}(L)}{\Theta_{0}^{2}(L)+\Lambda_{0}^{2}(L)}
$$

and

$$
\Theta_{1}(L)=\frac{\Theta_{0}(L)}{\Theta_{0}^{2}(L)+\Lambda_{0}^{2}(L)}
$$

The initial Fresnel ratio $\Lambda_{0}(L)$ and the initial phase curvature $\Theta_{0}(L)$ are given by

$$
\Lambda_{0}(L)=\frac{2 L}{k w_{0}^{2}}
$$

and

$$
\Theta_{0}(L)=1-\frac{L}{F_{0}}
$$

For all calculation, value for $\mathrm{F}_{\mathrm{o}}=\infty$, is refer to collimated. As a diffuser is added at the transmitter part and we can varies the value of correlation length, hence the output beam parameters also varies. 


\subsection{Turbulence scintillation index}

For moderate to strong turbulence scintillation index is as above:

$$
\sigma_{I, \text { strong }}^{2}=\exp \left\{\frac{0.49 \sigma_{R}^{2}}{\left[1+0.56\left(1+\Theta_{e d}\right) \sigma_{R}^{12 / 5}\right]^{7 / 6}}+\frac{0.51 \sigma_{R}^{2}}{\left(1+0.69 \sigma_{R}^{12 / 5}\right)^{5 / 6}}\right\}-1
$$

\subsection{Log intensity}

Meanwhile the log intensity due to small-scale eddies is given by

$$
\sigma_{l n y}^{2}(D)=\frac{1.27 \sigma_{I}^{2} n_{y}^{-5 / 6}}{1+\frac{0.4 n_{y}}{\Lambda_{1}+\Omega}}
$$

where the corresponding cutoff frequency is

$$
n_{y}=3\left(\frac{\sigma_{I}}{\sigma_{B}}\right)^{12 / 5}\left(1+0.69 \sigma_{B}^{12 / 5}\right)
$$

Therefore the total log intensity due to large-scale and small-scale is

$$
\sigma_{I}^{2}(D)=\exp \left[\sigma_{\ln x}^{2}(D)+\sigma_{\ln y}^{2}(D)\right]-1
$$

\section{Result and discussion}

Fig. 2 shows the degradation of scintillation index when using the diffuser for strong turbulence. As we can see the scintillation index increase when the distance propagation increase. At short distance $2 \mathrm{~km}$, the scintillation index for coherent beam is 1.5 but with using the diffuser the scintillation index can be reduce to 1.2. However the effective of diffuser is not continuous where from the graph as we can see, above the distance propagation $3 \mathrm{~km}$ it will turn to flat value. The effect of diffuser is more greatly effect in weak turbulence regime [18].

Fig. 3 illustrates the reduction of average intensity signal due to diffuser. The coherent beam (without diffuser) has a peak amplitude 0.045 at $1.4 \mathrm{~km}$ but the partially coherent beam (with diffuser) at $0.8 \mathrm{~km}$. This is because the diffuser will expand the spot size beam and consequently reduce the mean intensity. Hence, as a result it reduce the power received at the receiver. However this effect can be reduce with using dual diffuser modulation approach where it can double up the magnitude power as shown in Fig. 4. As example at $3 \mathrm{~km}$ distance propagation, the received power for coherent beam is $-6.6 \mathrm{dBm}$ and for conventional OOK using diffuser is $-7.6 \mathrm{dBm}$ but in diffusers modulation approach the received power is $-4.59 \mathrm{dBm}$. This show the improvement approximately 40 percent. 


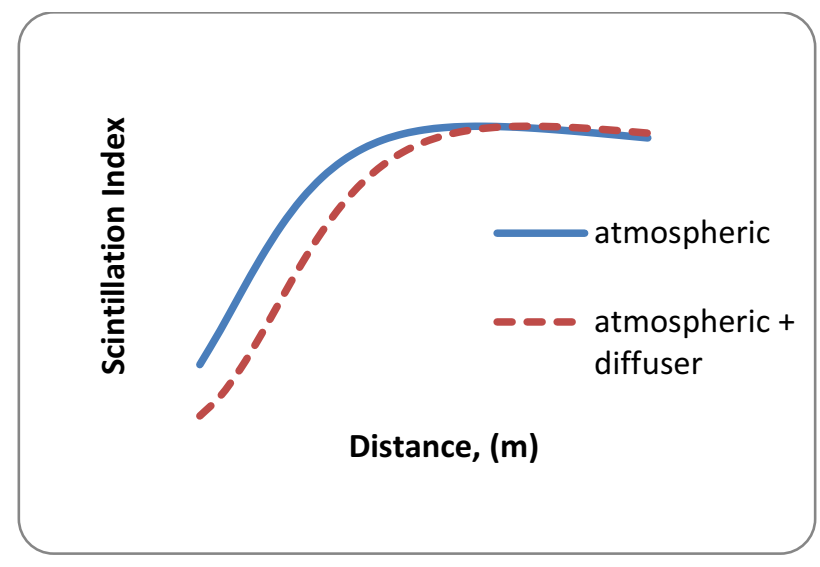

Fig. 2. Scintillation index versus distance for strong turbulence

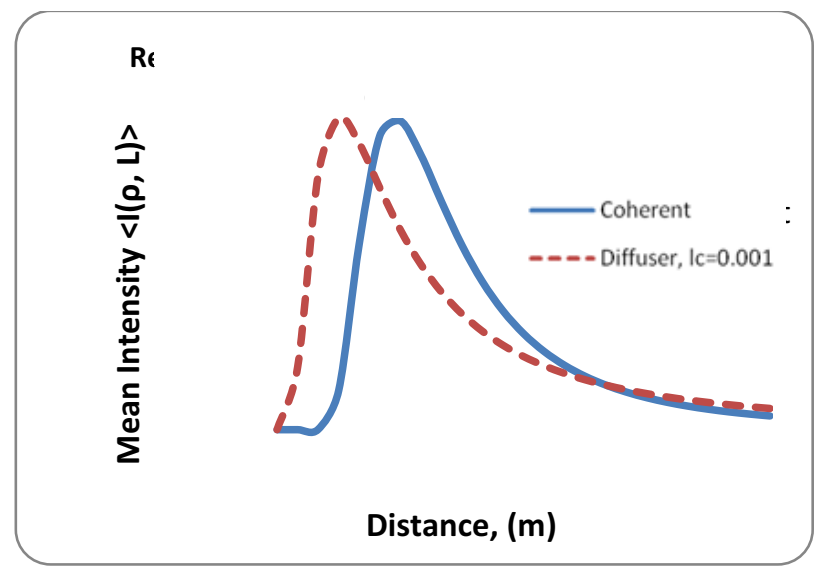

Fig. 3. Reduction of average intensity signal due to diffuser

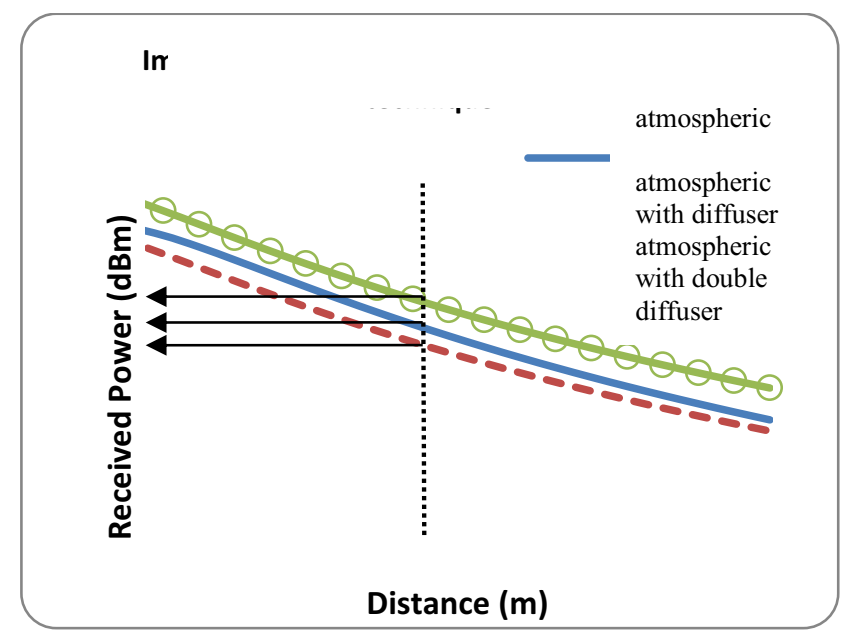

Fig. 4. Improvement of received power in Diffusers Modulation approach 


\section{Conclusion}

In contribution for this paper, the diffusers modulation improve the performance of the free space optic communication in atmospheric turbulence channel. The BER in atmospheric are strongly influenced by scintillation index, power received and signal technique which employed the partially coherent beam method improved the power received up to 40 percent and BER distance 42 percent longer compared to conventional OOK. As a result, it create the robust modulation against the turbulence with fix zero threshold value.

\section{References}

1. I.I. Kim, R. Stieger, J.A. Koontz, C. Moursund, M. Barclay, P. Adhikari, J. Schuster, E. Korevaar, R. Ruigrok, C. DeCusatis, Opt. Eng., 37, 3143 (1998)

2. S. Trisno, I. Smolyaninov, S.D. Milnerb, C.C. Davis, Optics \& Photonics 2005, (International Society for Optics and Photonics, 589215, 2005)

3. N. Namazi, R. Burris, G. C.Gilbreath, Optics \& Photonics 2005, (International Society for Optics and Photonics, 58920T, 2005)

4. M.C.R. Cordeiro, C.P. Colvero, J.P. von der Weid, International Conference on Microwave and Optoelectronics, IEEE, 393 (2005)

5. J. Li, M. Uysal, Global Telecommunications Conference, IEEE, 5, 2654 (2003)

6. I.B. Djordjevic, B. Vasic, M.A. Neifeld, IEEE Photon. Technol. Lett., 18, 1491 (2006)

7. M. Uysal, J. Li, M. Yu, IEEE Trans. Wireless Commun., 5, 1229 (2006)

8. M. Tariq, Avionics, Fiber-Optics and Photonics Technology Conference, IEEE, 21 (2007)

9. X. Zhu, J.M. Kahn, IEEE Trans. Commun., 50, 1293 (2002)

10. V.G. Sidorovich, V.V. Ragulsky, M.V. Vasil'ev, A.A. Leshchev, M.A. Sadovnikov, High-Power Lasers and Applications, International Society for Optics and Photonics, 191 (2002)

11. J.C. Ricklin, F.M. Davidson, J. Opt. Soc. Am. A, 19, 1794 (2002)

12. T. Shirai, A. Dogariu, E. Wolf, Directionality of some model beams propagating in atmospheric turbulence (Optics Letters, submitted).

13. G. Gbur, E. Wolf, JOSA A, 19, 8 (2002)

14. P. Raj, S. Sharma, P.C.S. Devara, J. Appl. Meteorol., 32, 1161 (1993)

15. L.C. Andrews, R.L. Phillips, C.Y. Hopen, Laser Beam Scintillation with Applications, (SPIE Press, Bellingham, 2001)

16. J.C. Ricklin, S.M. Hammel, F.D. Eaton, Atmospheric channel effectson free-space laser communication, (SpringerScience, 2007)

17. H.E. Nistazakis, T.A. Tsiftsis, G.S. Tombras, IET Commun. 3, 1402 (2009)

18. L.C. Andrews, R.L. Phillips, Laser Beam Propagation through Random Media, 2nd ed. (Bellingham, WA: SPIE Press., 2005)

19. O. Korotkova, L.C. Andrews, R. L. Phillips, Speckle propagation through atmosphere: effects of a random phase screen at the source (Proc. SPIE, 4821, 2000) 